\title{
Specification of regret-based models of choice behaviour: formal analyses and experimental design based evidence
}

\author{
Soora Rasouli ${ }^{1} \cdot$ Harry Timmermans ${ }^{1}$
}

Published online: 27 July 2016

(C) The Author(s) 2016. This article is published with open access at Springerlink.com

\begin{abstract}
Travel behaviour analysis has recently witnessed a rapidly growing interest in regret-based models of choice behaviour. Two different model specifications have been introduced in the transportation literature. Chorus et al. (Transportation Research B 42: 1-18, 2008a; in: Proceedings 87th Annual Meeting of the Transportation Research Board, Washington DC, 2008b) specified regret as a (non) linear function of the difference between the best-foregone choice alternative and the chosen alternative. Later, as an approximation to the original specification, Chorus (2010) suggested a logarithm function of utility differences between all choice alternatives, mainly for ease of estimation. This paper makes two contributions to this literature. First, formal analyses are conducted to identify the parameter space where the logarithmic specification becomes theoretically inferior to the original specification. Second, an empirical stated choice study on the choice of shopping centre is conducted to empirically test which specification best describes stated choices. Results suggest that for the collected data the original specification outperforms the new specification. Implications of this finding for the application of regret-based choice models in travel behaviour analysis are discussed.
\end{abstract}

Keywords Regret based models - Shopping centre choice - Stated choice experiment

\section{Introduction: background and problem statement}

Recently, regret-based choice models have gained rapidly in popularity in travel behaviour research as an alternative approach to modelling choice behaviour both under conditions of certainty and uncertainty. Seminal regret theory postulates that an individuals' regret

Soora Rasouli

s.rasouli@tue.nl

1 Urban Planning Group, Department of Urban Science and Systems, Eindhoven University of Technology, P.O. Box 513, 5600MB Eindhoven, The Netherlands 
derived from a binary decision is not only based on the anticipated payoff or utility of the considered choice alternative (across different states of the world), but also on anticipated payoff of the other alternative. More specifically, individuals are assumed to consider the possibility that the non-chosen alternative turns out to have a higher payoff than the chosen one after all. Regret occurs when an individual experiences or realizes that a higher payoff would have been achieved if the foregone alternative had been chosen. Thus, individuals are assumed not only to consider the attractiveness of the chosen alternative but also to anticipate the regret of not choosing the foregone alternative.

Regret-based models have been introduced in the travel behaviour community as an alternative approach to modelling travel decisions under conditions of uncertainty (Chorus et al. 2008a, b). The equivalent of random utility theory for decisions under conditions of uncertainty is expected utility theory, which assumes that individuals choose the choice alternative that will generate the highest expected pay-off, which is equal to the payoff of each decision outcome, multiplied by the probability of its occurrence. The violation of this property in many experiments in a variety of decision sciences has led to the formulation of alternative theories of risky choice and decision-making. (Cumulative) prospect theory (Kahneman and Tversky 1979; Tversky and Kahneman 1974, 1981, 1992) received most attention. It assumes that individuals frame possible outcomes in terms of gains and losses, relative to some neutral reference point, evaluate outcomes according to some value function and transform objective probabilities into subjective probabilities according to a non-linear probability weighting function. To account for violations of classic expected utility theory, the utility function is assumed concave over gains and convex over losses.

A recent overview of these studies on prospect theory is given in Li and Hensher (2011), while Rasouli and Timmermans (2014) provide a more extensive overview that also includes competing theories and modelling approaches. The majority of these studies concerned departure time and route choice decisions. For example, respondents in a study on route choice were asked to choose between two routes from work to home, which differed in terms of uncertain travel times, and estimated corresponding choice probabilities using cumulative prospect theory. They found evidence of violations of expected utility theory. In particular, they found evidence of the certainty effects and the inflation of small probabilities effect. Gao et al. (2010), comparing different approaches to model route choice behaviour, found that cumulative prospect theory outperformed expected utility theory, while Michea and Polak (2006) reached a similar conclusion in the context of the choice between two train services. Senbil and Kitamura (2004) found evidence in support of prospect theory in the context of departure time choice. Li et al. (2012) developed a nonlinear model accounting for both risk attitude and preference in the context of variable travel time. Their results suggest that the non-linear model better predicts earlier departure times compared to the linear model. Although most available research has focused on departure time and route choice, transport mode choice under risky situations has also been studied (Li and Hensher 2012; Li et al. 2013).

Regret-minimization theory constitutes an alternative to both expected utility and prospect theory. In the context of choice under conditions of certainty, regret-based models can be viewed as an alternative to IIA (independence of irrelevant alternative) models, such as the multinomial logit model, in the sense that choices are assumed to depend on choice set composition. Regretbased models allow incorporating substitution effects in an easy and elegant way.

Seminal work on regret theory for binary choices can be found in Bell (1982); Fishburn (1982); and Loomes and Sugden (1982). Chorus et al. (Chorus et al. 2006) and Chorus, Arentze and Timmermans (2008a, b), introducing regret-based choice models to the travel behaviour community, expanded this seminal work in two important ways. First, the model 
of binary choice was generalized to the choice among multiple (risky) choice alternatives. Second, the case of a single attribute was generalized to the case of multiple attributes. The extension to multiple choice alternatives was based on Quiggin's (1994) principle of Irrelevance of Statewise Dominated Alternatives, which states that a choice from any given choice set is not affected by adding or removing an alternative that is inferior for every state of the world. It implies that regret only depends on the best available choice alternative. The extension to multiple attributes was based on the contention that choice is based on a valuation of choice alternatives on an attribute-by-attribute basis. Let $n \in N$ represent the set of choice alternatives. Then, the linear regret function for attribute $k$ for choice alternative $n$ can be expressed as:

$$
\psi_{n n^{\prime} k}=\max \left[0, \beta_{k}\left(x_{k}^{n^{\prime}}-x_{k}^{n}\right)\right]
$$

Total regret when choosing alternative $n$ is then equal to

$$
R_{n}=\max _{n^{\prime}} \sum_{k=1}^{K} \psi_{n n^{\prime} k}
$$

Evidently, the linear regret function is a straightforward formulation, but it may not sufficiently capture the magnitude of regret and its relationship with attribute differences. Therefore, Chorus, Arentze and Timmermans (2008b) added a parameter to capture any non-linearities in regret.

If regret is defined in terms of not only the attribute difference between alternative $n$ and the best-foregone alternative, but against all available alternatives, Eq. 1 is generalized to:

$$
\psi_{n k}=\sum_{n^{\prime} \neq n \in C} \max \left[0, \beta_{k}\left(x_{k}^{n^{\prime}}-x_{k}^{n}\right)\right]
$$

Regret across all attributes can then be expressed as:

$$
R_{n}=\sum_{n^{\prime} \neq n \in C} \sum_{k=1}^{K} \max \left[0, \beta_{k}\left(x_{k}^{n^{\prime}}-x_{k}^{n}\right)\right]
$$

Defining regret as the utility difference against the best foregone choice alternative or as the sum of utility differences against all available alternatives has strong ramifications in terms of choice probabilities and the effects of introducing new alternatives to the choice set. As the focus of the current paper is empirical in nature, we leave these theoretical discussions for future publications.

Regret theory assumes that individuals minimize regret. Thus, alternative $n$ will be chosen if $R_{n}<R_{n^{\prime}} \forall n^{\prime} \neq n \in C$. Assuming that regret represents a random variable and that its error terms are independently Gumbel distributed, the probability of choosing alternative $n$ then equals

$$
p_{n}=\frac{\exp \left(-R_{n}\right)}{\sum_{n^{\prime}} \exp \left(-R_{n^{\prime}}\right)}
$$

A technical problem of this specification (which we will refer to as the original regret model specification) is that the max operators imply a non-smooth likelihood function, which may create problems in deriving marginal effects and elasticities. Therefore, 
(Chorus 2010 - see also Hensher et al. 2013; Chorus and Bierlaire 2013) suggested an alternative logarithmic specification, which we will refer to as the new regret model specification in the remaining of this paper. It can be expressed as

$$
R_{n}=\sum_{n^{\prime} \neq n \in C} \sum_{k=1}^{K} \ln \left(1+\exp \left(\beta_{k}\left(x_{k}^{n^{\prime}}-x_{k}^{n}\right)\right)\right)
$$

The number of studies using this specification is rapidly emerging in different disciplines (e.g., Beck et al. 2013; de Bekker-Grob and Chorus 2013; Chorus et al. 2013). Note that the new regret model specification differs from the original specification in two respects. Firstly, the discontinuous regret function is replaced with a continuous logarithmic function of attribute differences. The constant " 1 " is added to avoid that the logarithmic function is undefined. In principle, the choice of " 1 " is arbitrary, and could be replaced with any (small) number or a parameter. However, as we will discuss later, this specification has some implications. Secondly, regret is not based on the difference against the best alternative only but against all choice alternatives.

These two different specifications of regret raise the question which model should ideally be applied. The new logarithmic regret model specification avoids some practical issues and is easy to estimate. However, this comes at the cost of being theoretically inferior to the original model specification. That is, the specification is theoretically no longer consistent with the concept of regret as the value of the regret function is positive even if the chosen alternative turns out to be better than the foregone choice alternative. That is, Eq. (6) does not approximate zero if attribute differences are small and/or the number of choice alternatives is large. Moreover, the new specification estimates regret to be higher than regret based on the original specification, due to the constant 1 in the formulation. This difference has a disproportionally high effect when attribute differences and/or $\beta$ are small, and a negligible effect if these differences are high. The two specifications are approximately identical under major attribute differences only.

The comparison against all alternatives rather than against the best foregone alternative is also debatable. On the one hand, it is reasonable to assume that if an individual could have chosen multiple alternatives that perform better that the chosen one, regret will be higher. On the other hand, considering multiple better choice alternatives will further reduce the choice probabilities of the concerned alternative, which may be counter-intuitive. At least, there may be some diminishing effect. Behaviourally, particularly in the context of departure time and route choice decisions, assessing regret against all choice alternatives is cognitively very demanding as the number of alternatives is daunting. Moreover, it is not immediately clear in this decision context how to deal with autoregressive and spatial autocorrelation effects that are due to overlapping routes. Including all better foregone routes may thus involve routes that individual travellers will never consider or be unaware of. In this study, therefore, we argue that the decision to define regret against the best foregone choice alternative or against all foregone choice alternatives is an empirical matter that deserves further investigation.

Thus, for real-world applications, the empirical question is whether these theoretical shortcomings of the new logarithmic regret model specification are negligible. If they are, then, the new regret model specification is a good, easy to apply proxy of the theoretically sounder original model specification. If these effects are non-negligible, however, the original model specification should preferably be used. Moreover, the assumption of regret valuation on the singly best alternative or on all (foregone) choice alternatives should be empirically examined. 
The aim of the present study is to address these issues. A stated choice experiment is designed to investigate the effects of the signalled theoretical shortcomings of the new regret model specification and provide evidence whether respondents compare their choice against the best alternative or against all alternatives. Because the results of stated choice experiments are highly dependent on the specification of the attribute range, first we conduct a formal theoretical analysis to identify the parameter space and attribute level differences where the two model specifications differ most. The stated choice experiment was constructed on the basis of the outcomes of this formal analysis to optimize the comparison of the regret model specifications.

The remainder of the paper is organized as follows. First, we describe the results of the formal theoretical analysis. Based on the results of this analysis, we describe the design of the stated choice experiment. After discussing sample characteristics, we present the results of the model estimations. We complete the paper by drawing conclusions and discussing implications of our findings for the application of regret-based models in travel behaviour analysis.

\section{Formal analysis}

Consider two choice alternatives $n$ and $n^{\prime}$ with attribute levels $x_{n}$ and $x_{n^{\prime}}$ For each attribute $k$, the valuing function for different values of $x_{n}$ has two regimes: assuming that $x$ is monotonically increasing and $\beta_{k}>0$, (i) the regret regime for which $x_{n^{\prime}}>x_{n}$, and the nonregret regime for which $x_{n} \geq x_{n^{\prime}}$. Equivalent conditions can be identified for monotonically decreasing attributes and/or $\beta_{k}<0$.

\section{Non-regret regime}

Assuming a linear valuing function, according to the original model specification, regret in this regime is equal to zero, or

$$
R_{n}^{0}=0
$$

while for the new model specification, it is equal to

$$
R_{n}=\ln \left[1+\exp \left(\beta_{k}\left\{x_{k}^{n^{\prime}}-x_{k}^{n}\right\}\right)\right]
$$

A boundary condition for this regime appears if $x_{n}=x_{n^{\prime}}$ Eq. (8) then becomes

$$
R_{n}=\ln [1+\exp (0)]=\ln (2)=0.693147
$$

Thus, even though theoretically regret is zero in this case, the new regret specification gives a positive value for regret. It should be noted that this value increases with an increasing number of attributes and with an increasing number of inferior alternatives in the choice set. The newly formulated regret model is ill-founded under these conditions.

\section{Regret regime}

Assuming a linear valuing function, in this regime, regret according to the original model specification is equal to 


$$
R_{n}^{0}=\beta_{k}\left(x_{k}^{n^{\prime}}-x_{k}^{n}\right)
$$

while for the new model specification, it is equal to

$$
R_{n}=\ln \left[1+\exp \left(\beta_{k}\left\{x_{k}^{n^{\prime}}-x_{k}^{n}\right\}\right)\right]
$$

Noting that

$$
\ln (y+z)=\ln (y(z / y+1)=\ln (y)+\ln (z / y+1)
$$

Then,

$$
\begin{aligned}
\ln \left[1+\exp \left(\beta_{k}\left\{x_{k}^{n^{\prime}}-x_{k}^{n}\right\}\right)\right] & =\ln (1)+\ln \left[\exp \left(\beta_{k}\left\{x_{k}^{n^{\prime}}-x_{k}^{n}\right\}\right)+1\right] \\
& =\ln \left[\exp \left(\beta_{k}\left\{x_{k}^{n^{\prime}}-x_{k}^{n}\right\}\right)+1\right]
\end{aligned}
$$

Similarly,

$$
\begin{aligned}
\ln \left[\exp \left(\beta_{k}\left\{x_{k}^{n^{\prime}}-x_{k}^{n}\right\}\right)+1\right] & =\ln \left[\exp \left(\beta_{k}\left\{x_{k}^{n^{\prime}}-x_{k}^{n}\right\}\right)\right]+\ln \left[1 / \exp \left(\beta_{k}\left\{x_{k}^{n^{\prime}}-x_{k}^{n}\right\}\right)+1\right] \\
& \left.=\beta_{k}\left\{x_{k}^{n^{\prime}}-x_{k}^{n}\right\}\right)+\ln \left[1 / \exp \left(\beta_{k}\left\{x_{k}^{n^{\prime}}-x_{k}^{n}\right\}\right)+1\right]
\end{aligned}
$$

The boundary condition for Eq. (14) happens when the denominator is equal to 1 , implying that the maximum difference between the two models would be $\ln$ $(2)=0.693147$. This difference will become higher with an increasing number of attributes. The difference may even further increase with an increasing choice set size if regret is defined as the summation of regret across all better foregone alternatives.

The curvature of the difference between the two regret function specifications can be expressed and examined by taking the first derivative of the difference between the new regret specification and the old specification: $\ln \left(1+\exp \left(\beta_{k}\left(x_{n^{\prime}}-x_{n}\right)\right)\right)-\beta_{k}\left(x_{n^{\prime}}-x_{n}\right)$

$$
\frac{\partial \text { Diff }}{\partial \beta_{k}}=\frac{\exp \left(\beta_{k}\left(x_{n^{\prime}}-x_{n}\right)\right) *\left(x_{n^{\prime}}-x_{n}\right)}{1+\exp \left(\beta_{k}\left(x_{n^{\prime}}-x_{n}\right)\right)}-\left(x_{n^{\prime}}-x_{n}\right)=\frac{-\left(x_{n^{\prime}}-x_{n}\right)}{1+\exp \left(\beta_{k}\left(x_{n^{\prime}}-x_{n}\right)\right)}
$$

Similarly:

$$
\frac{\partial \text { Diff }}{\partial\left(x_{n^{\prime}}-x_{n}\right)}=\frac{\exp \left(\beta_{k}\left(x_{n^{\prime}}-x_{n}\right)\right) * \beta_{k}}{1+\exp \left(\beta_{k}\left(x_{n^{\prime}}-x_{n}\right)\right)}-\beta_{k}
$$

or, by re-arranging

$$
\frac{\partial \operatorname{Diff}}{\partial\left(x_{n^{\prime}}-x_{n}\right)}=\frac{-\beta_{k}}{1+\exp \left(\beta_{k}\left(x_{n^{\prime}}-x_{n}\right)\right)}
$$

Equations (16) and (18) clearly show that the difference between two regret formulations increases with decreasing $\beta_{\mathrm{k}}$ and $\left(x_{n^{\prime}}-x_{n}\right)$.

To illustrate the effects of the added constant in specifying regret from its true magnitude under different values of $\beta$ and different attribute differences, two hypothetical alternatives with one attribute, the values of which range from zero to one hundred with a 10 -point interval, were assumed. $\beta$ was varied across 4 different values: $0.01,0.1,0.5$ and 
1. Figure 1 visualizes the difference between the credible regret obtained by the original regret formulation and the new regret model specification. It suggests that the difference ranges between 0.6931 and 0 with the maximum at the lowest $\beta=0.01$ and minimum difference between attribute levels. It goes without saying that with an increasing number of attributes, this error tends to increase.

\section{Empirical study}

\section{Design of experiment}

The goal of the analysis is to (i) empirically assess the ramifications of the theoretical incongruence of the new logarithmic regret formulation specification with the notion of regret, and (ii) to compare the performance of the new regret formulation against the original regret model specification. To that end, a stated choice experiment was designed. The context was shopping destination choice.

To allow testing the basic assumptions underlying the new specification of the regret model, while keeping the experiment simple, we selected three attributes that were systematically varied in the experiment: travel time, number of stores and parking fees. Four levels were selected for each attribute. Attribute levels were chosen such as to make the design most sensitive to possible limitations of the newly formulated regret model, while keeping the task realistic. As our theoretical analysis has shown that the new regret model specification may fail when attribute differences are small and there is an expected tradeoff between attributes, the following attribute levels were defined. The number of stores was varied between 50, 52, 54 and 56 stores. Levels defined for parking fees were 0.50 , 1.00 and 1.50 and 2 Euros. Travel time was varied between 10, 20, 30 and $40 \mathrm{~min}$.

To permit examining the assumption of regret against the best foregone alternative only versus regret against all alternatives, three destinations were included in the experiment: Shopping Centre A, B and C. Attributes were varied independently both within and

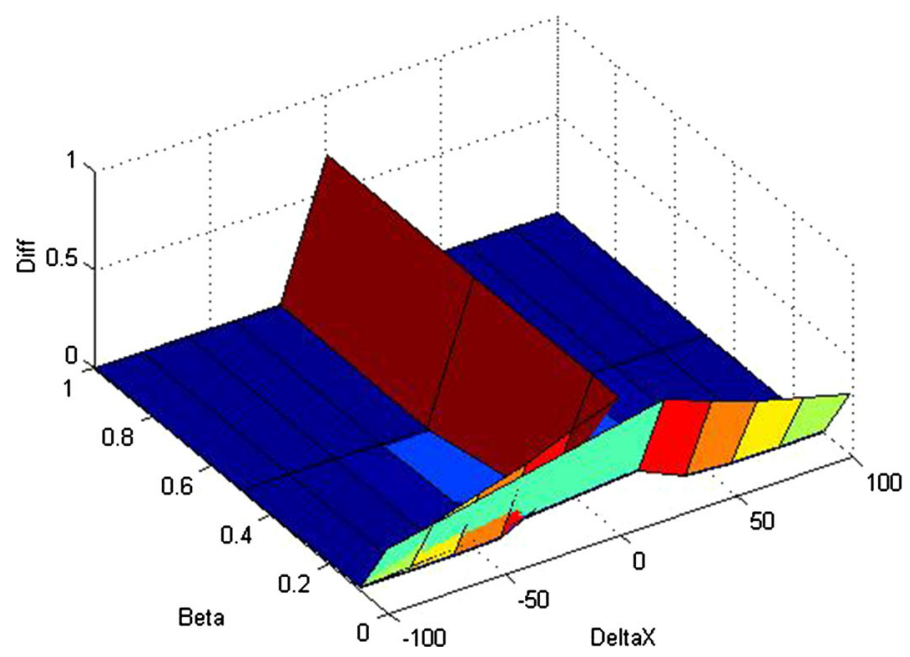

Fig. 1 Original versus new specification of regret 
between shopping centres. Hence, a $4^{9}$ orthogonal fractional factorial design consisting of 128 runs was created. An orthogonal fractional factorial design was chosen to satisfy the condition of attribute balance and because the alternative of constructing an optimal design tends to increase attribute level differences which is exactly what we wanted to avoid.

The orthogonal fractional factorial design was created such as to block the experiment into 16 orthogonal subsets. Consequently, the blocks are independent of all attributes. It implies that any response bias will not systematically affect the evaluation and choice of attribute profiles varied in the blocks. Each run thus consists of a choice set of three shopping centres (A, B and C) that vary systematically in terms of number of stores (size), parking fees and distance. Respondents were invited to choose the shopping centre they liked best from each choice set. Choice sets were randomized across respondents.

\section{Administration}

The experiment, complemented with questions about socio-demographics, was administered via Pauline, a platform for the creation and administration of Web-based questionnaires, developed by our group. A commercial firm was hired to recruit respondents. The chosen firm maintains a panel with known socio-demographics, which can be used to select or filter out particular segments of the population. This study only recruited respondents older than 18 years of age. Our sample was a random sample of the panel, which in turn is, according to the data collection firm, representative of the Dutch population. Although we have some doubts about such claims in general, the representativeness of the sample is not an issue here as generalization to the Dutch population is not an aim of this study.

The target sample size was 500 respondents. Data collection started on Monday, July 15,2013 . The target sample size was achieved the next day. This efficacy is due to the fact that panellists have committed to completing questionnaires quickly. Privacy of respondents was assured by setting up a connection between the firm and our Web application, filtering out their email addresses. Ultimately, 525 valid questionnaires were obtained.

\section{Sample characteristics}

Table 1 reports the frequency distributions of the selected socio-demographic characteristics. It shows that $49.5 \%$ of the sample is male, implying $50.5 \%$ is female. Age was classified into six categories. The percentages for these categories are respectively 11.6, $18.4,28.9,29.8,10.1$ and $1.1 \%$, indicating that most age categories are well represented in the sample, except the elderly ( $>75$ years old). It emphasizes that even if the panel would be representative of the Dutch population, the ultimate sample used for analysis is not. Hence, we do not make any claims regarding the generalizability of the results.

Table 1 further shows that households with one or two members constitute $63 \%$ of the sample. Singles and married respondents without children represent $65 \%$. Looking at work status reveals that a large percentage works part time. For education, results show that the middle and higher education level is overrepresented in the sample. 
Table 1 Frequency distribution of socio-demographic characteristics

\begin{tabular}{|c|c|c|c|}
\hline & Percentage & & Percentage \\
\hline Gender & & Household size & \\
\hline Male & 49.5 & & \\
\hline Female & 50.5 & 1 & 20.1 \\
\hline Age & & 2 & 42.8 \\
\hline $18-25$ & 11.6 & 3 & 15.6 \\
\hline $26-35$ & 18.4 & 4 & 14.6 \\
\hline $36-50$ & 28.9 & 5 & 6.4 \\
\hline $51-65$ & 29.8 & 6 & 0.4 \\
\hline $66-75$ & 10.1 & 8 & 0.1 \\
\hline$>75$ & 1.1 & Income & \\
\hline Marital status & & $<650$ Euro & 9.8 \\
\hline Single & 28.0 & $650 \ll 1250$ & 22.5 \\
\hline Couples without children & 37.0 & $1251 \ll 1875$ & 29.8 \\
\hline Couples children $<12$ & 15.2 & $1876 \ll 2500$ & 23.7 \\
\hline Couples children $>12$ & 19.8 & $>2500$ & 14.2 \\
\hline Education & & Paid work & \\
\hline Elementary school & 1.5 & & \\
\hline Lower vocational school & 8.1 & $0-10$ & 35.9 \\
\hline Middle general education & 13.7 & $11-20$ & 10.6 \\
\hline Middle specialized education & 7.9 & $21-30$ & 10.3 \\
\hline Middle vocational education & 32.3 & $31-40$ & 41.5 \\
\hline Higher vocational education & 25.6 & $41-50$ & 1.5 \\
\hline University & 10.9 & $51-60$ & 0.2 \\
\hline
\end{tabular}

\section{Analyses and results}

As we have discussed, the difference between the new and original regret specification can be broken down into the functional form of the regret function (max vs. ln), and whether regret is based on the best foregone alternative only or against all alternatives. Therefore, the predictive performance of three different model specifications was compared: (i) the original regret model, which specifies regret in terms of the attribute difference with the best foregone alternative, (ii) a specification which specifies regret in terms of attributes differences against all dominant alternatives, and (iii) the new logarithmic regret model specification. In the remainder of the paper, let RRmax denote the original model specification in which regret is judged against the best alternative for each attribute separately. Let RRsum denote the specification that defines regret as the max utility differences between the chosen alternative and all foregone alternatives that yield a higher utility on the attribute of interest. Finally, let RRLog represent the new regret specification based on the logarithm function and all pairwise comparisons. Mathematical expressions for the various estimated model are as follows:

$$
\text { RRMax }=\max _{n^{\prime} \neq n \in C} \sum_{k=1}^{K} \max \left[0, \beta_{k}\left\{x_{k}^{n^{\prime}}-x_{k}^{n}\right\}\right]
$$




$$
\begin{gathered}
\text { RRSum }=\sum_{n^{\prime} \neq n \in C} \sum_{k=1}^{K} \max \left[0, \beta_{k}\left\{x_{k}^{n^{\prime}}-x_{k}^{n}\right\}\right] \\
R R \log =\sum_{n^{\prime} \neq n \in C} \sum_{k=1}^{K} \ln \left[1+\exp \left(\beta_{k}\left\{x_{k}^{n^{\prime}}-x_{k}^{n}\right\}\right)\right]
\end{gathered}
$$

To estimate the models, attribute levels were effect-coded. The independent variables of each regret model consist of the total regret of the three attributes varied in the experiments plus the effect-coded socio-demographic variables. This means that for $L$ attribute levels, $L-1$ indicator variables were constructed. Each attribute level is coded 1 on the corresponding indicator variable and 0 on all other indicator variables. The last level of each attribute was coded as -1 on all indicator variables.

The RRLog model was estimated with the latest version of NLOGIT, which has the possibility of estimating the new regret specification. The RRSum model was estimated by pre-processing to incorporate the outcome of "max operator" in the maximum likelihood calculation, while dedicated software was written to estimate the RRMax model.

The alternative model specifications were compared in terms of the likelihood value of the models, the percentage of correct predictions and pseudo R-squared. The results are listed in Table 2. Columns two to five show the estimated coefficients, their standard errors and $P$ values for the three attributes of the hypothetical shopping centres: number of stores, Parking Fees and Travel Time. Columns six to eight list the log-likelihood of the estimated models, the corresponding percentage of correct predictions and pseudo R-squared. The final three columns show the observed and predicted market shares for all three shopping centres.

Because the various regret models differ in terms of the specification of the regret function and in terms of the number of compared choice alternatives, the discussion of the results focuses on these two aspects. Before presenting the results, note that Table 2 shows that the signs of the estimated coefficients of all models are all in anticipated directions. All estimated coefficients are statistically significant at the $5 \%$ probability level. When interpreting differences, it should be emphasized from the outset that the specifications of the regret functions are (almost) identical for a substantial segment of the attribute space. Thus, overall differences in model performance and predictive validity are expected to be small.

Table 2 shows that, as expected, parameters have the same sign in all three model specifications. Parking fees is the most influential attribute in all models. Comparing RRSum and RRLog shows that number of stores has a higher positive value, while parking fees has a more negative value in RRLog. The negative effect of distance is higher for RRSum. Because the regret models based on the pairwise comparison of alternatives are sensitive to choice set size, direct comparison of the parameter values of these two model types and RRMax is not meaningful. Therefore, to compare estimated parameters between these three models, the relative importance of one attribute relative to another attribute was calculated. To do so, it is assumed that distance in one alternative is increased by $1 \mathrm{~km}$. Regret increases 0.149 units in RRMax compared to other alternatives.

Table 3 provides the number of units decrease/increase required for the other attributes to create the same increase in regret subject to one unit increase in distance. Table 3 suggests that the effects of the number of stores and parking fees are the highest in RRLog compared to the other two regret models. To reach the same increase in regret, the number of stores needs to be reduced by 3.38 units, while parking fees needs to be increased by 


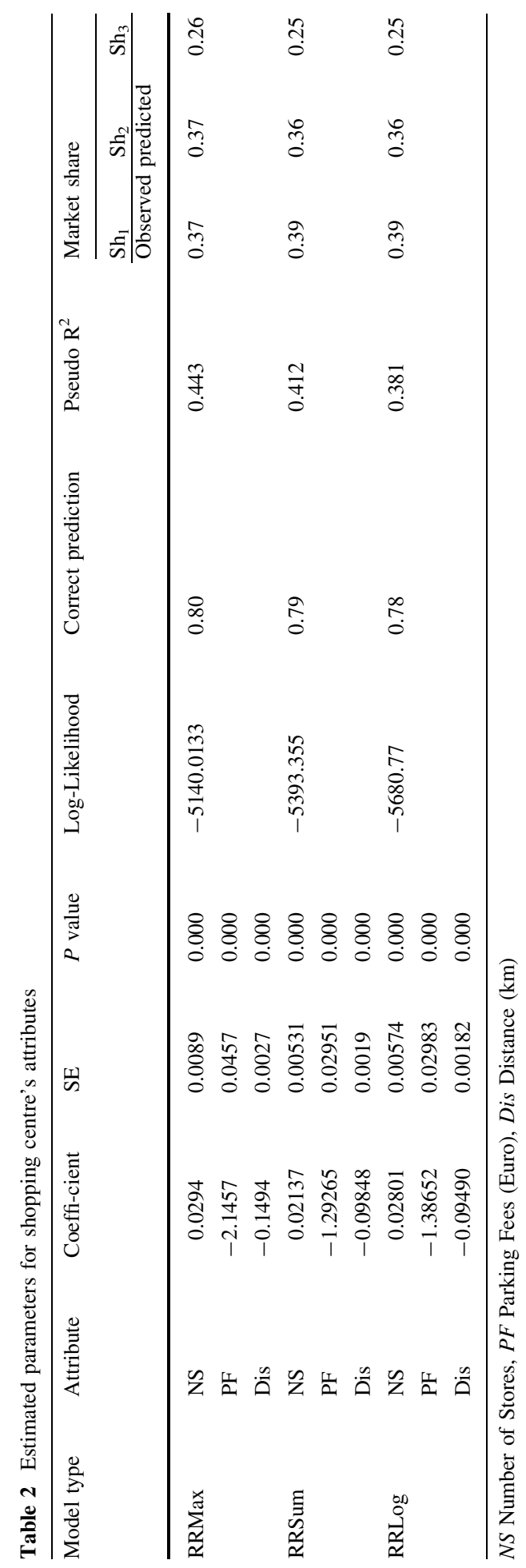


Table 3 Relative importance of attributes in three models

\begin{tabular}{lccc}
\hline & RRMax & RRsum & RRLog \\
\hline NS & -5.08 & -4.608 & -3.388 \\
PF & 0.0696 & 0.0762 & 0.0684 \\
\hline
\end{tabular}

Table 4 Elasticity of attributes in three models

\begin{tabular}{lccc}
\hline & RRMax & RRSum & RRLog \\
\hline Dist & -0.55 & -3.11 & -2.18 \\
NS & 0.45 & 1.058 & 0.716 \\
PF & -0.3619 & -2.027 & -1.48 \\
\hline
\end{tabular}

0.0684 units in RRLog. To obtain the same regret for RRMax and RRSum more units need to be deducted for the number of stores (5.08 and 4.608), while more units need to be added to parking fees (0.0696 and 0.0762).

Mean elasticities of the three attributes obtained for all three models are summarized in Table 4. It shows that for the RRsum specification that the probability of choosing the alternative increases $1.058 \%$ with an increase of the number of stores of one unit, while this increase is lower for RRMax $(0.45 \%)$ and RRLog $(0.716 \%)$. Likewise, when parking fees go up one unit, the RRsum model shows the highest drop in choice probabilities $(2.027 \%)$.

In order to investigate the significance of these elasticity differences, a KolmogorovSmirnov test was conducted. The results are reported in Table 5. The differences in elasticity of all attributes turn out to be significant at the $95 \%$ confidence level; the only exception is the difference between RRLog and RRSum corresponding to parking fees, which is non-significant.

Table 6 reports the estimated parameters for the socio-demographic variables for the three model specifications. It shows that most parameters are not significant. Exceptions include the 66-75 years age group, single persons, household size and number of working hours per week. Marginal utility is positive for all these 4 variables.

Coming back to Table 2, the Log-likelihood and pseudo R-squared related to RRMax, -4895.789 and 0.467 respectively-, are the highest of the 3 model specifications, suggesting that the original model specification best describes the current data. The second best model is RRSum with a Log-likelihood value of -5393.35 and a pseudo R-squared of 0.412 . The percentages correct predictions are 80 and $79 \%$ respectively. The worst model for the present data set is the new logarithmic model specification (RRLog) with a Loglikelihood of -5680.77 and pseudo $\mathrm{R}^{2}$ of 0.381 . The percentage correct predictions associated with this new model specification is also lower (78 \%). Based on the Ben-Akiva Swait test (1986) for non-nested models, the differences are significant. The predicted market shares for the three stores are identical for RRSum and RRLog with values of 39, 36 and $25 \%$ respectively. These values only slightly differ for RRMax: 37, 37 and $26 \%$.

Thus, Table 2 suggests that the best model in terms of goodness of fit and predictive success for the current data is the original regret specification, in which regret is judged against the best foregone alternative (RRMax). This finding implies that, at least in the present study, the principle of estimating regret across all superior forgone alternatives is not as satisfactory as the principle of regret over the best non-chosen alternative. Thus, the 


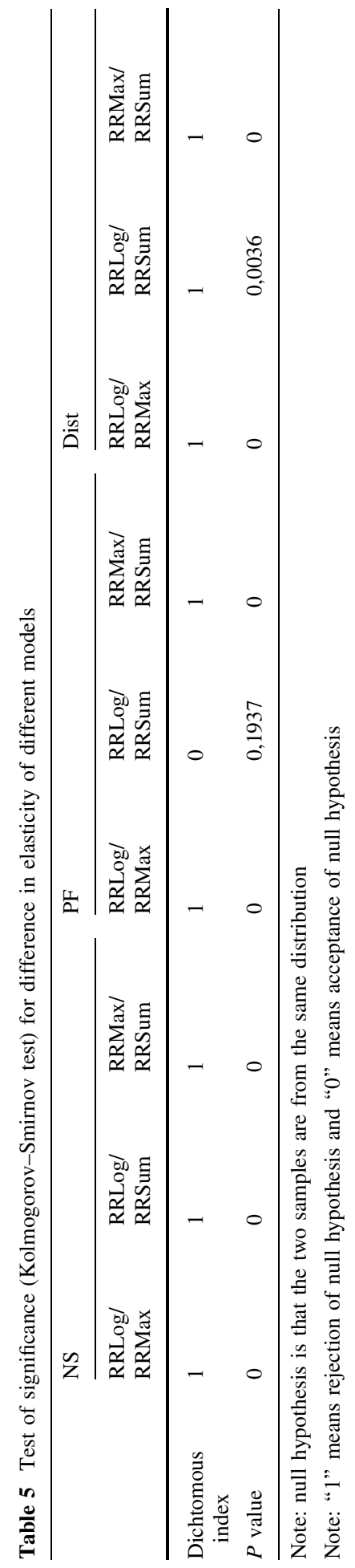




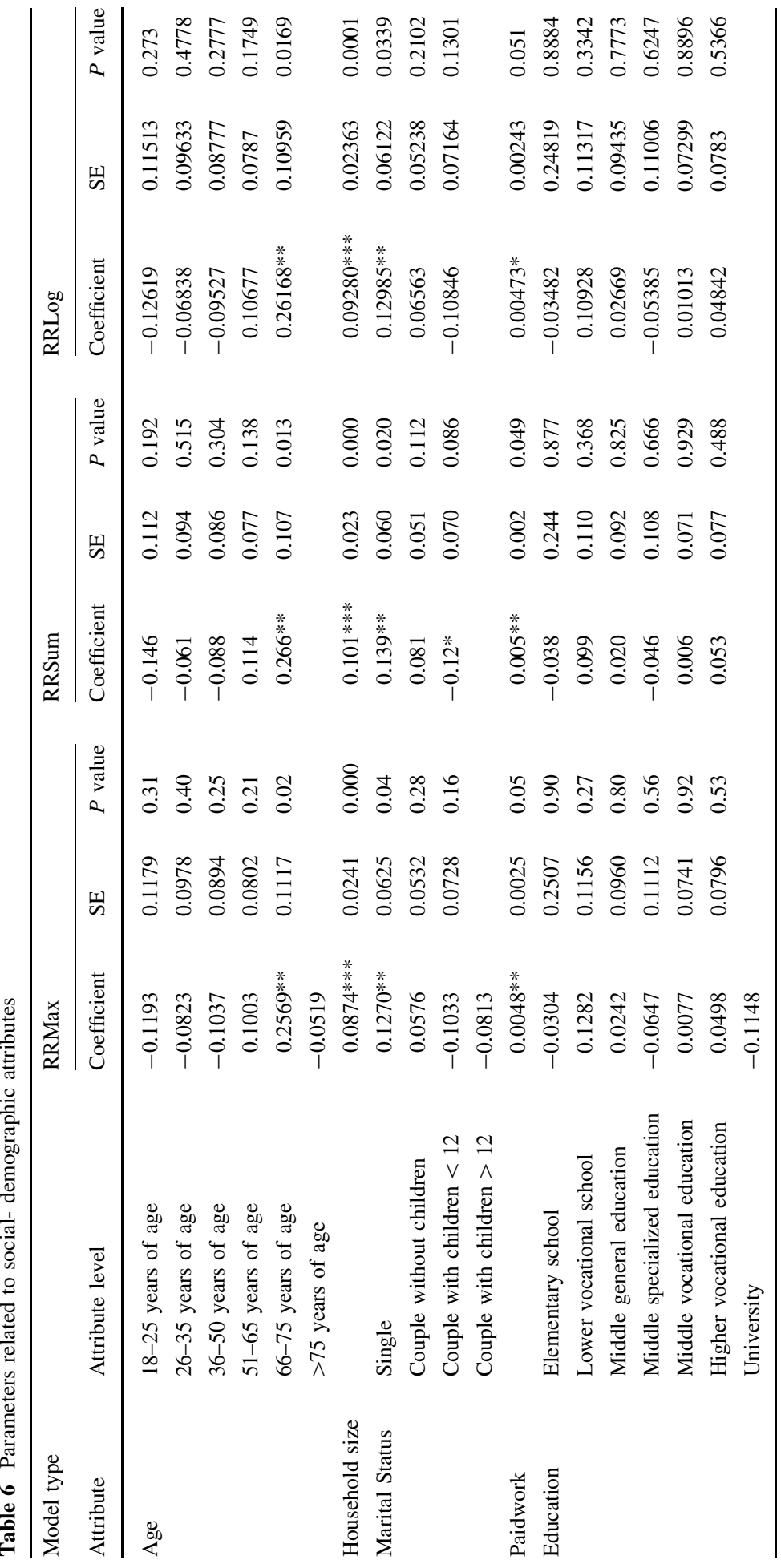




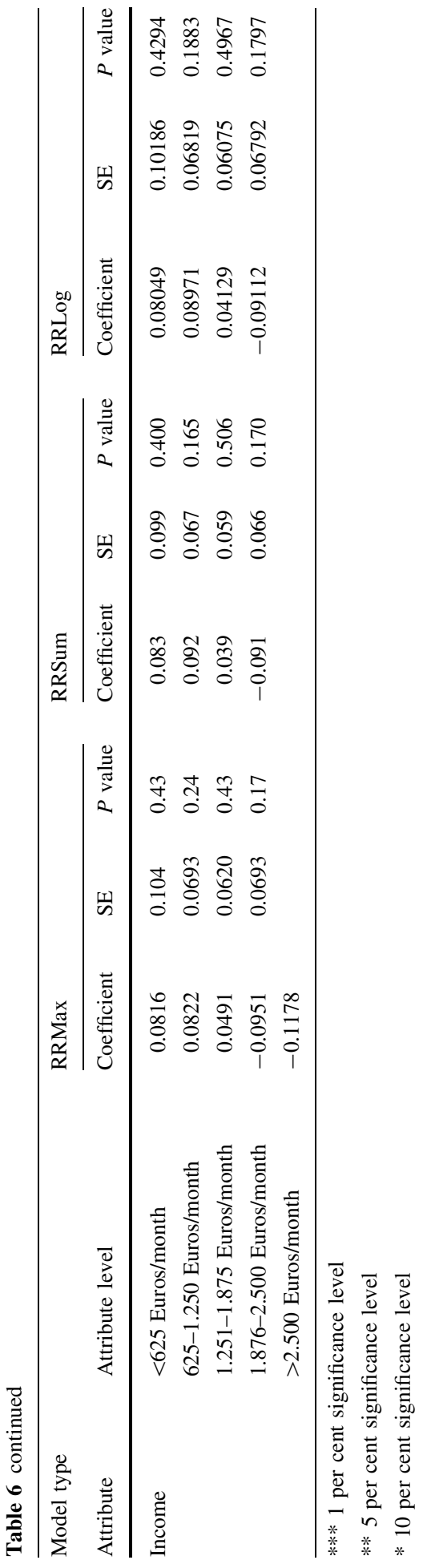


empirical findings in this study support the theoretical superiority of the original regret specification. If this result can be replicated in other studies, it seems that individuals do not assess regret by pairwise comparing multiple choice alternatives.

As for the functional form, the finding that the original regret function, summed across all better alternatives, outperforms the new logarithmic regret specification suggests that the theoretical incongruence with the notion of regret is reflected in these less accurate results. The new logarithmic specification, which was meant to approximate the original formulation, will result in substantial differences in choice probabilities if attribute differences are small. It performs less than the original specification, based on max operators, on the current data set that involves small attribute differences.

As we have mentioned from the outset, the original and new regret specifications are asymptotically identical for much of the attribute space. Consequently, the aggregate performance of the various regret models can at best show small differences. To further explore the performance differences between the three models, their predictive accuracy was examined separately for the 128 shopping centre profiles that were included in the experimental design. We expect this analysis to be more sensitive to the differences between the model specifications, considering we constructed the experiment such as to allow better discrimination between the models.

A summary of the results is provided in Table 7. Further more detailed results are presented in Table 8. As shown, RRMax better predicts 13 profiles than either RRSum or RRLog. This number increases to 18 when RRMax is compared with RRLog only. RRSum outperforms RRMax for 4 profiles only, and this number drops to 0 when a comparison is made with both RRMax and RRLog. There is no profile for which RRLog (the new logarithmic specification) performs better than both RRMax and RRsum.

Table 8 presents the profiles for which the three regret model specifications result in different predictions. Table 9 shows the observed market shares for the 3 shopping centres. The definition of these profiles is presented in Table 10. In many cases where RRMax predicts more accurately than the other regret specifications, the reason seems the inclusion of the constant in the regret function in RRLog, which has the effect of adding more regret in terms of number of stores and parking fees and calculating regret by summation over all outperformed alternatives (RRLog and RRsum). Some blocks clearly show the different predictive success of the models.

For block2-Cset13, RRMax predicts observed choices $18.5 \%$ more accurately than the two other regret models. Table 8 reveals that RRMax predicts that shopping centre one will invariably be chosen. This shopping centre is the closest one $(10 \mathrm{~km})$ but has less stores (52) and higher parking fees (2 Euro) compared to shopping centres 2 and 3 with 54 stores

Table 7 Summary performance of the three regret model specifications: Number of profiles more accurately predicted by different models

\begin{tabular}{|c|c|c|c|c|}
\hline $\begin{array}{l}\text { RRMax » } \\
\text { RRSum \& } \\
\text { RRLog }\end{array}$ & $\begin{array}{l}\text { RRMax } \gg \\
\text { RRSum }\end{array}$ & $\begin{array}{l}\text { RRMax } \gg \\
\text { RRLog }\end{array}$ & $\begin{array}{l}\text { RRSum » } \\
\text { RRMax \& } \\
\text { RRLog }\end{array}$ & $\begin{array}{l}\text { RRSum } \gg \\
\text { RRMax }\end{array}$ \\
\hline 14 & 14 & 19 & 1 & 5 \\
\hline RRsum $\gg$ RRLog & $\begin{array}{l}\text { RRLog } \gg \text { RRMax \& } \\
\text { RRSum }\end{array}$ & RRLog $\gg$ RRMax & RRLog $\gg$ RRSum & \\
\hline 9 & 0 & 4 & 2 & \\
\hline
\end{tabular}

$\gg$ outperform 
Table 8 Detailed comparison of profiles with different predictions by the models

\begin{tabular}{|c|c|c|c|c|c|c|c|c|c|c|c|c|}
\hline & \multicolumn{4}{|c|}{ RRMax } & \multicolumn{4}{|c|}{ RRLog } & \multicolumn{4}{|c|}{ RRSum } \\
\hline & \multicolumn{3}{|c|}{ Market share } & \multirow{2}{*}{$\begin{array}{l}\text { Correct } \\
(\%)\end{array}$} & \multicolumn{3}{|c|}{ Market share } & \multirow{2}{*}{$\begin{array}{l}\text { Correct } \\
(\%)\end{array}$} & \multicolumn{3}{|c|}{ Market share } & \multirow{2}{*}{$\begin{array}{l}\text { Correc } \\
(\%)\end{array}$} \\
\hline & $\mathrm{sh}_{1}$ & $\mathrm{sh}_{2}$ & $\mathrm{sh}_{3}$ & & $\mathrm{sh}_{1}$ & $\mathrm{sh}_{2}$ & $\mathrm{sh}_{3}$ & & $\mathrm{sh}_{1}$ & $\mathrm{sh}_{2}$ & $\mathrm{sh}_{3}$ & \\
\hline block0-Cset1 & 0 & 0 & 100 & 77.3 & 0 & 25.8 & 74.2 & 60.6 & 0 & 1.5 & 98.5 & 75.8 \\
\hline block0-Cset2 & 0 & 89.4 & 10.6 & 30.3 & 0 & 97 & 3 & 25.8 & 0 & 93.9 & 6.1 & 28.8 \\
\hline block0-Cset16 & 0 & 0 & 100 & 87.9 & 15.2 & 0 & 84.8 & 75.8 & 0 & 0 & 100 & 87.9 \\
\hline block1-Cset5 & 0 & 93.9 & 6.1 & 13.6 & 0 & 100 & 0 & 10.6 & 0 & 100 & 0 & 10.6 \\
\hline block1-Cset6 & 93.9 & 0 & 6.1 & 25.8 & 100 & 0 & 0 & 19.7 & 100 & 0 & 0 & 19.7 \\
\hline block1-Cset14 & 0 & 0 & 100 & 83.3 & 7.6 & 0 & 92.4 & 78.8 & 0 & 0 & 100 & 83.3 \\
\hline block2-Cset13 & 100 & 0 & 0 & 58.5 & 0 & 100 & 0 & 40 & 0 & 100 & 0 & 40 \\
\hline block2-Cset15 & 0 & 100 & 0 & 52.3 & 0 & 10.8 & 89.2 & 20 & 0 & 10.8 & 89.2 & 20 \\
\hline block3-Cset1 & 0 & 100 & 0 & 55.4 & 0 & 87.7 & 12.3 & 52.3 & 0 & 87.7 & 12.3 & 52.3 \\
\hline block3-Cset3 & 0 & 0 & 100 & 44.6 & 95.4 & 0 & 4.6 & 36.9 & 78.5 & 0 & 21.5 & 44.6 \\
\hline block4-Cset1 & 0 & 0 & 100 & 78.1 & 51.6 & 0 & 48.4 & 45.3 & 14.1 & 0 & 85.9 & 70.3 \\
\hline block4-Cset6 & 100 & 0 & 0 & 70.3 & 75 & 0 & 25 & 57.8 & 54.7 & 0 & 45.3 & 51.6 \\
\hline block4-Cset15 & 0 & 0 & 100 & 82.8 & 0 & 15.6 & 84.4 & 67.2 & 0 & 1.6 & 98.4 & 81.3 \\
\hline block5-Cset4 & 33.3 & 0 & 66.7 & 43.9 & 100 & 0 & 0 & 62.1 & 100 & 0 & 0 & 62.1 \\
\hline block5-Cset9 & 0 & 100 & 0 & 27.3 & 100 & 0 & 0 & 59.1 & 100 & 0 & 0 & 59.1 \\
\hline block6-Cset2 & 89.4 & 0 & 10.6 & 43.9 & 98.5 & 0 & 1.5 & 40.9 & 98.5 & 0 & 1.5 & 40.9 \\
\hline block6-Cset3 & 0 & 100 & 0 & 30.3 & 100 & 0 & 0 & 69.7 & 100 & 0 & 0 & 69.7 \\
\hline block6-Cset5 & 0 & 0 & 100 & 51.5 & 0 & 36.4 & 63.6 & 56.1 & 0 & 36.4 & 63.6 & 56.1 \\
\hline block6-Cset7 & 30.3 & 0 & 96.7 & 63.6 & 100 & 0 & 0 & 27.3 & 100 & 0 & 0 & 27.3 \\
\hline block6-Cset14 & 0 & 100 & 0 & 71.2 & 0 & 77.3 & 22.7 & 63.6 & 0 & 51.5 & 48.5 & 50 \\
\hline block6-Cset16 & 0 & 1.5 & 98.5 & 98.4 & 0 & 0 & 100 & 68.2 & 0 & 0 & 100 & 68.2 \\
\hline block7-Cset6 & 0 & 0 & 100 & 86.6 & 0 & 4.5 & 95.5 & 82.1 & 0 & 0 & 100 & 86.6 \\
\hline block7-Cset13 & 0 & 1.5 & 98.5 & 79.1 & 10.4 & 0 & 89.6 & 73.1 & 0 & 0 & 100 & 80.6 \\
\hline
\end{tabular}

and 0.5 Euro, and 52 stores and 1 Euro respectively. In contrast, RRLog and RRSum predict that the second shopping centre, which is much further than shopping centre 1 $(40 \mathrm{~km})$ but outperforms shopping centre 1 on the two other attributes, will be chosen. This clearly shows that adding the constant in the RRLog model and summing regret over all outperformed attributes in the RRLog and RRSum models decreases the accuracy of prediction.

RRMax predicts $32 \%$ better than the two other models for block2-Cset 15 . In this case, RRMax predicts that all respondents would go to shopping centre 2 because it is the closest even though it has the highest parking fees (10 km and 2 Euro). Table 9 proves that actually more than half of people choose this alternative. In contrast, RRLog and RRSum predict that only $11 \%$ of the respondents would choose shopping centre 2 . In turn, these models predict that almost $90 \%$ will select shopping centre 3, which is much further $(30 \mathrm{~km})$, but has a parking fee that is 1 Euro cheaper than the fee of shopping centre 2 . In this case, RRSum estimates high regret because of the summation of regret for parking fees for shopping centre 2. RRLog overweights regret for parking fees due to the constant.

The same reasoning holds for block6-Cset7. While the empirical data in Table 9 indicate that the market share of shopping centre 3 , which is the closest one $(20 \mathrm{~km})$ with 
Table 9 Observed market share

\begin{tabular}{|c|c|c|c|c|c|c|c|}
\hline & \multicolumn{7}{|c|}{ Observed market share } \\
\hline & $\mathrm{sh}_{1}$ & $\mathrm{sh}_{2}$ & $\mathrm{sh}_{3}$ & & $\mathrm{sh}_{1}$ & $\mathrm{sh}_{2}$ & $\mathrm{sh}_{3}$ \\
\hline block0-Cset1 & 10.6 & 12.1 & 77.3 & block4-Cset15 & 3.1 & 14.1 & 82.8 \\
\hline block0-Cset2 & 4.5 & 22.7 & 72.8 & block5-Cset4 & 62.2 & 4.5 & 33.3 \\
\hline block0-Cset16 & 10.6 & 1.5 & 87.9 & block5-Cset9 & 59.1 & 27.3 & 13.6 \\
\hline block1-Cset5 & 40.7 & 10.5 & 48.8 & block6-Cset2 & 39.4 & 6.1 & 54.5 \\
\hline block1-Cset6 & 19.7 & 18.2 & 62.1 & block6-Cset3 & 69.7 & 30.3 & 0 \\
\hline block1-Cset14 & 7.6 & 9.1 & 83.3 & block6-Cset5 & 4.6 & 43.9 & 51.5 \\
\hline block2-Cset13 & 58.5 & 40 & 1.5 & block6-Cset7 & 27.3 & 9.1 & 63.6 \\
\hline block2-Cset15 & 30.8 & 52.3 & 16.9 & block6-Cset14 & 4.5 & 71.2 & 24.3 \\
\hline block3-Cset1 & 12.3 & 55.4 & 32.3 & block6-Cset16 & 10.6 & 21.2 & 68.2 \\
\hline block3-Cset3 & 50.8 & 4.6 & 44.6 & block7-Cset6 & 3 & 10.4 & 86.6 \\
\hline block4-Cset1 & 17.2 & 4.7 & 78.1 & block7-Cset13 & 4.5 & 14.9 & 80.6 \\
\hline block4-Cset6 & 70.3 & 14.1 & 15.6 & & & & \\
\hline
\end{tabular}

$20 \mathrm{~km}$ difference to the 2 other alternatives, is $64 \%$, RRLog ad RRSum predict that all respondents choose shopping centre 1 because this alternative has more stores (56) than shopping centre 3 and parking fees are one Euro cheaper (1 Euro). The predictive accuracy of these two models is $36 \%$ less than the accuracy of RRMax.

There are two profiles that RRSum and RRLog predict considerably better than RRMax. The first choice set is (block5-Cset9), in which RRMax predicts that all respondents choose the closest shopping centre $2(10 \mathrm{~km})$. The difference is that in this choice set distance is not as superior as in the other cases where the distance to the best alternative was 20 or $30 \mathrm{~km}$ less than to the other alternatives. Rivalling shopping centre 1, which the two other models predict to be chosen, is $20 \mathrm{~km}$ away, which is only $10 \mathrm{~km}$ further than the distance to shopping centre 2 . It outperforms the other centre on the two other attributes (i.e., more stores (56 against 52) and lower parking fee (1 Euro against 1.5 Euro).

Block6-Cset3 is another profile that RRMax predicts less accurately than the other two regret models. In this case, RRMax predicts that all travellers choose shopping centre 2, which has the best performance of all alternatives in terms of the number of stores and parking fees (56 and 0.5 Euro) but is located $10 \mathrm{~km}$ further away than the closest alternative, which is shopping centre 1 .

In summary, results demonstrate that in situations where one alternative is superior over the two others with respect to distance (more than $10 \mathrm{~km}$ closer) RRMax generally more accurately predicts observed destination choice behaviour than the two other regret models. In turn, in choice sets where the superior alternative with respect to distance is only $10 \mathrm{~km}$ closer, RRMax predicts similar or less accurate than RRLog and RRSum.

\section{Conclusions and discussion}

Transportation researchers have recently embraced regret-based models of travel choice as an viable alternative to utility-maximizing models. Regret-based models have been formulated for both decision-making under certainty and decision-making under uncertainty. They offer a potentially powerful alternative to the multinomial logit model and the 
Table 10 Definition of profiles

\begin{tabular}{|c|c|c|c|c|c|c|c|c|c|}
\hline & \multicolumn{3}{|c|}{ Shopping centre 1} & \multicolumn{3}{|c|}{ Shopping centre 2} & \multicolumn{3}{|c|}{ Shopping centre 3} \\
\hline & NS & $\mathrm{PF}$ & Dis & NS & $\mathrm{PF}$ & Dis & NS & $\mathrm{PF}$ & Dis \\
\hline block0-Cset1 & 52 & 1 & 40 & 54 & 1.5 & 20 & 50 & 1.5 & 10 \\
\hline block0-Cset2 & 56 & 2 & 20 & 54 & 1 & 10 & 56 & 0.5 & 10 \\
\hline block0-Cset16 & 54 & 1 & 20 & 50 & 2 & 40 & 52 & 1 & 10 \\
\hline block1-Cset5 & 50 & 1.5 & 40 & 50 & 2 & 30 & 52 & 2 & 30 \\
\hline block1-Cset6 & 54 & 2 & 20 & 54 & 1.5 & 40 & 56 & 2 & 20 \\
\hline block1-Cset14 & 54 & 1.5 & 20 & 50 & 1 & 40 & 54 & 1.5 & 10 \\
\hline block2-Cset13 & 52 & 2 & 10 & 54 & 0.5 & 40 & 52 & 1 & 40 \\
\hline block2-Cset15 & 56 & 0.5 & 40 & 54 & 2 & 10 & 54 & 1 & 30 \\
\hline block3-Cset1 & 50 & 1.5 & 30 & 56 & 2 & 10 & 52 & 1.5 & 20 \\
\hline block3-Cset3 & 54 & 1.5 & 10 & 56 & 1.5 & 30 & 54 & 0.5 & 20 \\
\hline block4-Cset1 & 52 & 1 & 30 & 56 & 1.5 & 40 & 50 & 0.5 & 30 \\
\hline block4-Cset6 & 50 & 2 & 10 & 54 & 1 & 40 & 52 & 0.5 & 40 \\
\hline block4-Cset15 & 50 & 2 & 30 & 56 & 1.5 & 30 & 52 & 1.5 & 20 \\
\hline block5-Cset4 & 56 & 1 & 30 & 50 & 1 & 30 & 56 & 1.5 & 20 \\
\hline block5-Cset9 & 56 & 1 & 20 & 52 & 1.5 & 10 & 54 & 0.5 & 40 \\
\hline block6-Cset2 & 50 & 0.5 & 10 & 52 & 1 & 20 & 52 & 0.5 & 10 \\
\hline block6-Cset3 & 50 & 1 & 20 & 56 & 0.5 & 30 & 56 & 1 & 40 \\
\hline block6-Cset5 & 52 & 2 & 20 & 50 & 1.5 & 10 & 54 & 0.5 & 20 \\
\hline block6-Cset7 & 56 & 1 & 40 & 52 & 1 & 40 & 52 & 2 & 20 \\
\hline block6-Cset14 & 56 & 1 & 40 & 50 & 2 & 10 & 52 & 0.5 & 40 \\
\hline block6-Cset16 & 52 & 2 & 10 & 52 & 0.5 & 30 & 50 & 1.5 & 10 \\
\hline block7-Cset6 & 52 & 1.5 & 30 & 52 & 1 & 20 & 52 & 1 & 10 \\
\hline block7-Cset13 & 56 & 1.5 & 30 & 52 & 0.5 & 40 & 54 & 1.5 & 20 \\
\hline
\end{tabular}

expected value model for choices where individuals may compromise. They are based on the principle that individuals do take into account the loss of utility, which they may experience due to the utility difference between the chosen and the foregone choice alternative(s).

At the start of this study, two different regret model specifications can be found in the transportation literature. The original specification is an extension of earlier work outside transportation and only considers regret with regard to the best, non-chosen alternative, specified as a non-continuous utility function. The second specification, included in NLOGIT, considers regret vis-à-vis all non-chosen choice alternatives, captured in terms of a continuous logarithmic regret function.

Focusing on the case of regret under conditions of certainty, the current paper first identifies and discusses some theoretical caveats related to the latter specification. Theoretical arguments, mathematical derivations and a numerical illustration are provided to explore the boundary conditions under which this specification becomes theoretically problematic. Beyond the theoretical debate, we argue it is critically important to compare the performance of the different model specifications using empirical data.

Thus, in addition to the theoretical arguments and numerical illustration, this paper reports the results of a stated choice experiment about the choice of shopping centre. The 
experiment was constructed in such a way that the design (definition of attribute levels) was highly sensitive to the differences between the regret models. The results of the mathematical derivations and numerical illustration showed that differences between the two model specifications increased for relatively small attribute differences and small parameter values. Therefore, the experimental design is based on small attribute differences for two out of the three experimentally varied attributes. Moreover, to empirically examine the validity of the hypothesis of regret, based on all foregone choice alternatives, versus regret, based on the best choice alternative only, the choice set consisted of three shopping centres in total.

Results of model estimations showed that the original model specification outperformed the new specification for the present data set, which is consistent with our theoretical arguments. This implies that the continuous logarithm formulation of regret seems less valid for when attribute differences are small. Of course, this paper only reported the results of a single study. It goes without saying that the current study should be replicated for other choice problems and in other study areas to confirm the current results. However, if the present findings would be substantiated in such replications, researchers should be aware of these differences. The new logarithmic specification of the regret model only approximates the original, model specification if attribute differences become larger. It should be realized that attribute differences in typical application domains such as route choice, transit service choice and departure time choice tend to be small in many cities. Hence, in some travel choice domains, the stipulated problems may not be trivial in the sense that the two specifications of the regret model may lead to substantial differences in predicted choice probabilities. The relative lack of fit may be caused by the arbitrarily added constant of 1 . Problems would be less if a smaller value had been chosen.

The results of the experiment with respect to the number of foregone choice alternatives to be included in the specification of the regret function suggest that the original regret model, which is based on the single best foregone alternative, appeared to be the most accurate model for the current data. To be able to better understand the behaviour of the two formulations in different situations, replications of this study to different choice problems are required.

The current study has only compared the performance of two regret models. Future research should extend the comparison and include yet other choice models. For example, models of attribute processing and attendance, hybrid models and the heterogeneous heuristic choice model (Zhu and Timmermans 2008, 2010) may be alternative choice models that better capture choice processes under the stipulated conditions.

The new logarithmic specification of regret turned out to perform the worst. Thus, it seems that individuals do not accumulate proportionally regret if they face multiple better performing choice alternatives on a specific attribute. In this context, it should be mentioned that the distinction between two or three choice alternatives may be minor. Higher reduction in prediction accuracy is expected by increasing the number of alternatives. In any case, it is also unrealistic to assume, for example in the context of route choice behaviour, that all routes are included in the regret function because individuals will not be familiar with all routes and routes are not independent. Thus, even though future research would indicate that regret may be based on more than the single best foregone choice alternative only, the current specification that is based on all pairwise comparisons is unrealistic for such complex choice problems with many alternatives. Moreover, the theoretical result that adding multiple inferior choice alternatives would change choice probabilities is also not very appealing. 
Although this study was primarily conducted to shed more light on the empirical performance of the two basic specifications of regret-based models of riskless choice behaviour, the findings suggest some interesting issues for future research that goes beyond these basic models. First, the current models assume that individuals are aware of all choice alternatives, but this assumption is not founded on empirical results. Thus, one line of research should consider which alternatives to include. Second, the issue how many choice alternatives to include in the calculation of regret also requires further study for more complex choice problems. In many domains, choice alternatives will be correlated and the research question is whether such correlation should be included in the specification of the regret function. Route choice behaviour is a clear example. It is unlikely that individuals would count separately all minor deviations from say the shortest route that they did not choose. In general, because regret-based models using multiple choice alternatives depend heavily on choice set composition, the effects of the definition of the choice set and predicted choice probabilities need more systematic study. Third, although that has not been the main focus of our analysis, the results of the current analysis show that modelling choice behaviour based on regret only has problems capturing clear tradeoffs in the decision-making process. More advanced choice models, based on multiple choice mechanisms should be developed. Finally, the current paper was concerned with choice behaviour under conditions of certainty. Similar studies should be conducted for regret models that predict decision making under conditions of uncertainty.

We hope that this paper will lead to many replications and applications of regret-based models in the travel behaviour community so that the community at large can access a body of accumulated knowledge that indicates which specification most likely gives the best results with a specific application domain in mind. We plan to continue this stream of work along the lines suggested.

Open Access This article is distributed under the terms of the Creative Commons Attribution 4.0 International License (http://creativecommons.org/licenses/by/4.0/), which permits unrestricted use, distribution, and reproduction in any medium, provided you give appropriate credit to the original author(s) and the source, provide a link to the Creative Commons license, and indicate if changes were made.

\section{References}

Beck, M.J., Chorus, C.G., Rose, J.M., Hensher, D.A.: Vehicle purchasing behaviour of individuals and groups: regret or reward? J. Transp. Econ. Policy. 47(3), 475-492 (2013)

Bell, D.E.: Regret in decision making under uncertainty. Oper. Res. 30(5), 961-981 (1982)

Chorus, C.G.: A new model of random regret minimization. Eur. J. Transp. Infrastruct. Res. 10, 181-196 (2010)

Chorus, C.G., Molin, E.J.E., Van Wee, B., Arentze, T.A., Timmermans, H.J.P.: Responses to transit information among car-drivers: regret-based models and simulations. J. Transp. Plan. Technol. 29, 249-271 (2006)

Chorus, C.G., Arentze, T.A., Timmermans, H.J.P.: A Random Regret-Minimization model of travel choice. Transp. Res. B 42, 1-18 (2008a)

Chorus, C.G., Arentze, T.A., Timmermans, H.J.P.: A comparison of regret minimization and utility-maximization in the context of travel mode-choices. In: Proceedings 87th Annual Meeting of the Transportation Research Board, Washington DC (2008b)

Chorus, C.G., Bierlaire, M.: Travel choice models that generate preferences for compromise alternatives: an empirical comparison. In: Proceedings 92nd Annual Meeting of the Transportation Research Board, Washington DC (2013)

Chorus, C.G., Koetse, M.J., Hoen, A.: Consumer preferences for alternative fuel vehicles: comparing a utility maximization and a regret minimization model. Energy Policy 61, 901-908 (2013) 
de Bekker-Grob, E.W., Chorus, C.G.: Random regret-based discrete choice modelling: an application to health care. PharmacoEconomics 31(7), 623-634 (2013)

Fishburn, P.C.: Non-transitive measurable utility. J. Math. Psychol. 26(1), 31-67 (1982)

Gao, S., Frejinger, E., Ben-Akiva, M.: Adaptive route choices in risky traffic networks: A prospect theory approach. Transp. Res. C 18, 727-740 (2010)

Hensher, D.A., Greene, W.H., Chorus, C.G.: Random regret minimization or random utility maximisation: An exploratory analysis in the context of automobile fuel choice. J. Adv. Transp. 47, 667-678 (2013)

Kahneman, D., Tversky, A.: Prospect theory: an analysis of decisions under risk. Econometrica 47(2), 263-291 (1979)

Li, Z., Hensher, D.A.: Prospect theoretic contributions in understanding traveler behaviour: a review and some comments. Transp. Policy 31, 97-115 (2011)

Li, Z., Hensher, D.A.: Accommodating risk attitudes in freight transport behaviour research. Transp. Rev. 32(2), 221-239 (2012)

Li, Z., Hensher, D.A., Rose, J.M.: Accommodating perceptual conditioning in the valuation of expected travel time savings for cars and public transport. Res. Transp. Econ. 39(1), 270-276 (2013)

Li, Z., Tirachini, A., Hensher, D.A.: Embedding risk attitudes in a scheduling model: application to the study of commuting departure time. Transp. Sci. 46(2), 170-188 (2012)

Loomes, G., Sugden, R.: Regret-theory: an alternative theory of rational choice under uncertainty. Econ. J. 92(368), 805-824 (1982)

Michea, A., Polak, J.: Modelling risky choice behaviour: evaluating alternatives to expected utility theory. Paper presented at the 11 th International Conference on Travel Behaviour Research, Kyoto, Japan (2006)

Quiggin, J.: Regret theory with general choice sets. J. Risk. Uncertain. 8(2), 153-165 (1994)

Rasouli, S., Timmermans, H.J.P.: Applications of theories and models of choice and decision-making under conditions of uncertainty in travel behavior research. Travel. Behav. Soc. 1(3), 79-90 (2014)

Senbil, M., Kitamura, R.: Reference points in commuter departure time choice: a prospect theoretic test of alternative decision frames. Intell. Transp. Syst. 8, 19-31 (2004)

Tversky, A., Kahneman, D.: Judgment under uncertainty: heuristics and biases. Science 185, 1127-1131 (1974)

Tversky, A., Kahneman, D.: The framing of decisions and the psychology of Choice. Science 211, 453-458 (1981)

Tversky, A., Kahneman, D.: Advances in prospect theory: cumulative representation of uncertainty. J. Risk. Uncertain. 9, 195-230 (1992)

Zhu, W., Timmermans, H.J.P.: Cut-off models for the 'go-home' decision of pedestrians in shopping streets. Environ. Plan. B 35, 248-260 (2008)

Zhu, W., Timmermans, H.J.P.: Cognitive process model of individual choice behaviour incorporating principles of bounded rationality and heterogeneous decision heuristics. Environ. Plan. 37, 59-74 (2010) 